\title{
CURVES OF CENTROIDS, GERGONNE POINTS AND SYMMEDIAN CENTERS IN TRIANGLE PENCILS IN ISOTROPIC PLANE
}

\author{
Mirela Katić Žlepalo and Ema JuRkin
}

\begin{abstract}
In this paper we consider a triangle pencil in an isotropic plane consisting of the triangles that have the same circumscribed circle. We study the curves of centroids, Gergonne points and symmedian centers for such pencils of triangles. We also study the curves of centroids, Gergonne points and symmedian centers for tangential triangles of such a triangle pencil.
\end{abstract}

\section{INTRODUCTION}

An isotropic plane is a projective-metric plane, where the absolute figure consists of a real line, called the absolute line and a real point, called the absolute point which lies on the absolute line, [6]. The distance between two non-parallel points $A=\left(x_{A}, y_{A}\right)$ and $B=\left(x_{B}, y_{B}\right), x_{A} \neq x_{B}$, is defined as $d(A, B)=x_{B}-x_{A}$. They have the midpoint $\left(\frac{1}{2}\left(x_{A}+x_{B}\right), \frac{1}{2}\left(y_{A}+y_{B}\right)\right)$. Two non-parallel lines $p$ and $q$ given by the equations $y=k_{p} x+l_{p}$ and $y=k_{q} x+l_{q}$, $k_{p} \neq k_{q}$, form an angle defined by $\angle(p, q)=k_{q}-k_{p}$. Therefore, their bisector is given by the equation $y=\frac{1}{2}\left(k_{p}+k_{q}\right) x+\frac{1}{2}\left(l_{p}+l_{q}\right)$.

It has been shown in [5] that each triangle $A B C$ in the isotropic plane can be set, by a suitable choice of the coordinate system, into the so-called standard position, in which its vertices are of the form $A=\left(a, a^{2}\right), B=\left(b, b^{2}\right)$, $C=\left(c, c^{2}\right)$, where $a+b+c=0$ and its circumscribed circle has the equation $y=x^{2}$. In the papers [1], [4] and [5], the authors proved that for the standard triangle in the isotropic plane the coordinates of the centroid are $G=\left(0,-\frac{2 q}{3}\right)$, of the Gergonne point $\Gamma=\left(-\frac{3 p}{q},-\frac{4 q}{3}\right)$ and of the symmedian center $K=\left(\frac{3 p}{2 q},-\frac{q}{3}\right)$, where $p=a b c$ and $q=b c+c a+a b$.

In this paper, we will consider the triangles in the isotropic plane:

2010 Mathematics Subject Classification. 51N25.

Key words and phrases. Isotropic plane, pencil of triangles, centroid, Gergonne point, symmedian center. 
- the pencil of triangles having the same circumscribed circle $y=x^{2}$,

- the tangential triangles of all the triangles in such a pencil.

We will prove that the locus of all centroids of all triangles having the same circumscribed circle is a circle, the locus of all Gergonne points is a curve of order 4 and the locus of all symmedian centers is an ellipse. For the tangential triangles of all the triangles in such a pencil we will prove that the locus of all centroids of all triangles is a line, the locus of all Gergonne points is an ellipse and the locus of all symmedian centers is a curve of order 3 .

\section{The PEnCIL of TRIANgles HAVING The SAME CIRCUMSCRIBED CIRCLE}

Let $A=\left(a, a^{2}\right), B=\left(b, b^{2}\right)$ and $C=\left(c, c^{2}\right)$ be the vertices of the triangle having the circumscribed circle $y=x^{2}$. The sides of the triangle $A B C$, i.e. the lines $A B, B C$ and $A C$ have the equations

$$
\begin{aligned}
& y=(a+b) x-a b, \\
& y=(b+c) x-b c, \\
& y=(a+c) x-a c,
\end{aligned}
$$

respectively. The tangent lines to the circle $y=x^{2}$ at the points $A, B$ and $C$ are given by equations:

$$
\begin{aligned}
& y=2 a x-a^{2}, \\
& y=2 b x-b^{2}, \\
& y=2 c x-c^{2} .
\end{aligned}
$$

The midpoint of the side $B C$ of the triangle is $A_{m}=\left(\frac{b+c}{2}, \frac{b^{2}+c^{2}}{2}\right)$ and the equation of the median $A A_{m}$ is

$$
y=\frac{2 a^{2}-b^{2}-c^{2}}{2 a-b-c} x+\frac{a\left(b^{2}-a b+c^{2}-a c\right)}{2 a-b-c} .
$$

Analogously, the equation of the median $B B_{m}$ is

$$
y=\frac{2 b^{2}-a^{2}-c^{2}}{2 b-a-c} x+\frac{b\left(a^{2}-a b+c^{2}-b c\right)}{2 b-a-c}
$$

and the equation of the median $C C_{m}$ is

$$
y=\frac{2 c^{2}-a^{2}-b^{2}}{2 c-a-b} x+\frac{c\left(a^{2}-a c+b^{2}-b c\right)}{2 c-a-b} .
$$

The intersection point $\left(\frac{a+b+c}{3}, \frac{a^{2}+b^{2}+c^{2}}{3}\right)$ of lines $(2.7),(2.8)$ and (2.9) is the centroid of such a triangle. Instead of observing one triangle, we will observe the whole pencil of triangles. Let the vertices $A$ and $B$ be fixed and let the vertex $C$ move on the circumscribed circle. We calculate the locus of 
all centroids of all triangles in such a pencil by eliminating $c$ from equations (2.7) and (2.8) (or (2.9)). The equation of that locus is

$$
9 x^{2}-6(a+b) x-3 y+2\left(a^{2}+a b+b^{2}\right)=0
$$

and it is clear that this is a circle in the isotropic plane (Figure 1). We have proved the following theorem:

THEOREM 2.1. The curve of centroids of all the triangles in the pencil of triangles having the same circumscribed circle is a circle in the isotropic plane.

Let us notice that the circle (2.10) intersects the line $A B$ in the points $\left(\frac{2 a+b}{3}, \frac{2 a^{2}+b^{2}}{3}\right)$ and $\left(\frac{a+2 b}{3}, \frac{a^{2}+2 b^{2}}{3}\right)$, i.e. the centroids of two degenerated triangles of the pencil, obtained when $C$ coincides with $A$ and $B$.

Next, in order to determine the Gergonne point of the triangle $A B C$, we should first calculate the equation of its inscribed circle. The inscribed circle has the equation

$$
y=\frac{1}{4} x^{2}+\frac{1}{2}(a+b+c) x+\frac{1}{4}\left(a^{2}+b^{2}+c^{2}-2 a b-2 a c-2 b c\right) .
$$

The points of tangency of the inscribed circle (2.11) and the sides (2.1), (2.2) and (2.3) are

$$
\begin{aligned}
& C_{g}=\left(-c+a+b, a^{2}+b^{2}-a c-b c+a b\right), \\
& A_{g}=\left(-a+b+c, b^{2}+c^{2}-a b-a c+b c\right), \\
& B_{g}=\left(-b+a+c, a^{2}+c^{2}-a b-b c+a c\right),
\end{aligned}
$$

respectively. The lines $A A_{g}, B B_{g}$ and $C C_{g}$ have the equations

$$
\begin{aligned}
& y=\frac{a^{2}-b^{2}-c^{2}+a b+a c-b c}{2 a-b-c} x+\frac{a\left(a^{2}+b^{2}+c^{2}-2 a b-2 a c+b c\right)}{2 a-b-c} \\
& y=\frac{b^{2}-a^{2}-c^{2}+a b+b c-a c}{2 b-a-c} x+\frac{b\left(a^{2}+b^{2}+c^{2}-2 a b-2 b c+a c\right)}{2 b-a-c} \\
& y=\frac{c^{2}-a^{2}-b^{2}+a c+b c-a b}{2 c-a-b} x+\frac{c\left(a^{2}+b^{2}+c^{2}-2 a c-2 b c+a b\right)}{2 c-a-b}
\end{aligned}
$$

respectively. The lines (2.12), (2.13) and (2.14) intersect in the Gergonne point of the triangle $A B C$. In order to get the pencil of the triangles, we will keep the vertices $A$ and $B$ fixed and let the vertex $C$ move along the circumscribed circle. The locus of all Gergonne points in such a triangle pencil is obtained by eliminating $c$ from any two of three equations (2.12), (2.13) and (2.14). The equation of that locus is 


$$
\begin{gathered}
4\left(a^{2}+a b+b^{2}\right) x^{4}-6(a+b) x^{3} y+3 x^{2} y^{2}-6\left(a^{3}+b^{3}\right) x^{3} \\
+\left(a^{2}-8 a b+b^{2}\right) x^{2} y+6(a+b) x y^{2}-3 y^{3}+3\left(3 a^{4}+2 a^{3} b-9 a^{2} b^{2}\right. \\
\left.+2 a b^{3}+3 b^{4}\right) x^{2}-6\left(2 a^{3}-3 a^{2} b-3 a b^{2}+2 b^{3}\right) x y+2\left(2 a^{2}-7 a b+2 b^{2}\right) y^{2} \\
-18 a b\left(a^{3}-a^{2} b-a b^{2}+b^{3}\right) x+3\left(4 a^{3} b-9 a^{2} b^{2}+4 a b^{3}\right) y+9 a^{2} b^{2}(a-b)^{2}=0
\end{gathered}
$$

i.e. a curve of order 4 (Figure 1). Therefore, we have proved the following theorem:

THEOREM 2.2. The curve of Gergonne points of all the triangles in the pencil of triangles having the same circumscribed circle is a curve of order 4 in the isotropic plane.

As a curve of order 4 , this curve has four intersections with the absolute line. Two of those intersections are conjugate-imaginary and the other two intersections coincide with the absolute point in which this curve touches the absolute line. So, it is a 2-circular quartic. Detailed studies of circular quartics in the isotropic plane were given in [2] and [3].

Furthermore, the curve of Gergonne points has cusps in the points $A$ and $B$, which correspond to the cases when the point $C$ coincides with the points $B$ and $A$, respectively. It also has a double point in the point $D=$ $\left(\frac{a+b}{2}, a^{2}-a b+b^{2}\right)$, which is the intersection point of the lines through $A$, $B$, parallel to the tangent lines at $B, A$ to the circumscribed circle $y=x^{2}$, respectively. The double point is obtained for the values $c=\frac{a+b \pm \sqrt{5}(b-a)}{2}$. (Note the connection with golden ratio $\frac{c-a}{b-a}=\frac{b-a}{c-b}$ !) According to [7], the curve of order 4 with two cusps and a double point is a rational curve of class 4.

The line

$$
y=\frac{a+3 b}{2}(x-a)+a^{2}
$$

is obviously the tangent to the curve of Gergonne points at the cusp $A$. It is parallel to the bisector of the line $A B$ given by (2.1) and the tangent to the circle $y=x^{2}$ at the point $B$ given by (2.5). Analogously, the tangent to the curve of Gergonne points at the cusp $B$ is parallel to the bisector of the line $A B$ and the tangent to the circle $y=x^{2}$ at the point $A$.

The tangents to the curve of Gergonne points at the double point $D$ are given by equations

$$
y=\left((a+b) \pm \frac{3 \sqrt{5}}{5}(b-a)\right)\left(x-\frac{a+b}{2}\right)+a^{2}-a b+b^{2} .
$$

Their bisector given by the equation

$$
y=(a+b) x+\frac{a^{2}-4 a b+b^{2}}{2}
$$


is parallel to the line $A B$.

Next, we will consider the symmedian centers. The angle bisector of the lines (2.1) and (2.3) with the vertex $A$ has the equation

$$
y=\frac{2 a+b+c}{2} x-\frac{a(b+c)}{2} .
$$

The angle with the vertex $A$ formed by the median (2.7) and the angle bisector (2.18) must be the same as the angle between the angle bisector (2.18) and the symmedian through the vertex $A$. It is easy to calculate that the equation of the symmedian through the vertex $A$ has the equation

$$
y=\frac{2\left(a^{2}-b c\right)}{2 a-b-c} x+\frac{2 a b c-a^{2} b-a^{2} c}{2 a-b-c} .
$$

Analogously, the other two symmedians may be calculated. All three of them intersect in the same point, so-called symmedian center. We will calculate the locus of all symmedian centers of all the triangles in the pencil, keeping the vertices $A$ and $B$ fixed and moving the vertex $C$ on the circumscribed circle. Eliminating $c$ from any two of three symmedian equations, we obtain the equation

$4\left(a^{2}+a b+b^{2}\right) x^{2}-6(a+b) x y+3 y^{2}-6 a b(a+b) x-\left(a^{2}-8 a b+b^{2}\right) y+3 a^{2} b^{2}=0$.

This locus is a conic and its intersections with the absolute line are conjugate-imaginary, therefore it is an ellipse (Figure 1). We have proved the following theorem:

THEOREM 2.3. The curve of symmedian centers of all the triangles in the pencil of triangles having the same circumscribed circle is an ellipse in the isotropic plane.

Some short calculations show that the coordinates of the points $A=$ $\left(a, a^{2}\right), B=\left(b, b^{2}\right)$ and $D=\left(\frac{a+b}{2}, a^{2}-a b+b^{2}\right)$ fulfill the equation (2.19). Thus, the curve of symmedian centers passes through all three singular points of the curve of Gergonne points. Furthermore, it touches the circumscribed circle at the fixed points $A$ and $B$, while its tangent line at the point $D$ is identical to the bisector given by (2.17), (Figure 1).

Next, we will calculate characteristic points of the tangential triangle of the given triangle $A B C$. The tangential triangle $A_{t} B_{t} C_{t}$ is a triangle formed by the tangent lines to the circumscribed circle of the given triangle $A B C$ at its vertices. The equations of the tangent lines are given in (2.4), (2.5), (2.6) and they intersect in the points $A_{t}=\left(\frac{b+c}{2}, b c\right), B_{t}=\left(\frac{a+c}{2}, a c\right)$ and $C_{t}=\left(\frac{a+b}{2}, a b\right)$.

Keeping the points $A$ and $B$ fixed and moving $C$ on the circle $y=x^{2}$, we obtain the pencil of tangential triangles $A_{t} B_{t} C_{t}$. It was proved in [1] that the Gergonne point of a tangential triangle is the symmedian point of its reference 


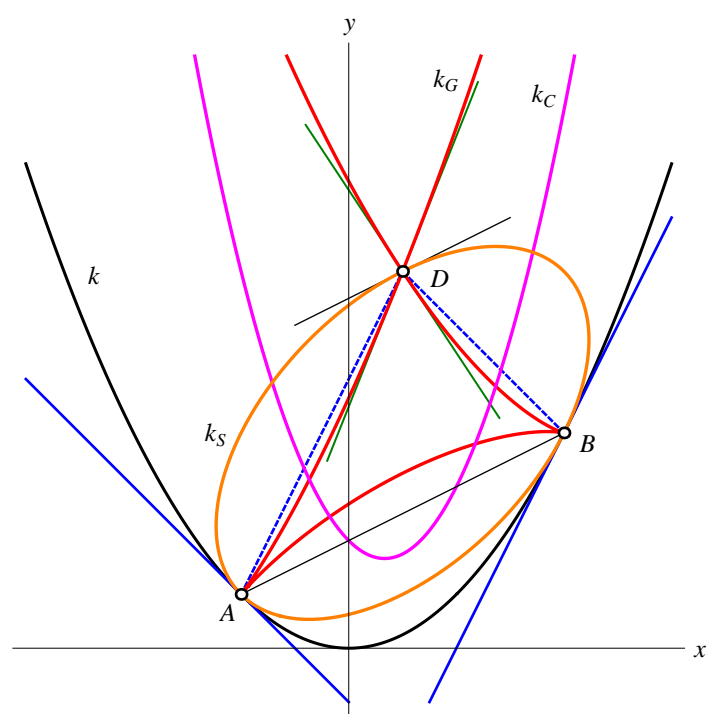

Figure 1. The curve of centroids $k_{C}$, curve of Gergonne points $k_{G}$ and curve of symmedian centers $k_{S}$ for a pencil of triangles with the same circumscribed circle $k$

triangle. Therefore, the locus of all Gergonne points of all tangential triangles in such a pencil is the curve given by (2.19), (Figure 2). Thus, we can state:

THEOREM 2.4. The curve of Gergonne points of all the tangential triangles in the pencil of triangles having the same circumscribed circle is an ellipse in the isotropic plane.

The midpoints of the sides $A_{t} B_{t}, A_{t} C_{t}$ and $B_{t} C_{t}$ are $C_{t m}=\left(\frac{a+b+2 c}{4}, \frac{a c+b c}{2}\right)$, $B_{t m}=\left(\frac{a+2 b+c}{4}, \frac{a b+b c}{2}\right)$ and $A_{t m}=\left(\frac{2 a+b+c}{4}, \frac{a b+a c}{2}\right)$. The medians $A_{t m} A_{m}$, $B_{t m} B_{m}$ and $C_{t m} C_{m}$ have the equations

$$
\begin{aligned}
& y=\frac{4 b c-2 a b-2 a c}{-2 a+b+c} x+\frac{\left(b^{2}+c^{2}\right) a-b c(b+c)}{-2 a+b+c}, \\
& y=\frac{4 a c-2 a b-2 b c}{a-2 b+c} x+\frac{\left(a^{2}+c^{2}\right) b-a c(a+c)}{a-2 b+c}, \\
& y=\frac{4 a b-2 a c-2 b c}{a+b-2 c} x+\frac{\left(a^{2}+b^{2}\right) c-a b(a+b)}{a+b-2 c},
\end{aligned}
$$

respectively. All three medians intersect in the centroid of the triangle $A_{t} B_{t} C_{t}$. Again, keeping the points of tangency $A$ and $B$ fixed and moving $C$ on the circle $y=x^{2}$, we obtain the pencil of tangential triangles $A_{t} B_{t} C_{t}$. 
Eliminating $c$ from any two of three equations (2.20), (2.21) and (2.22), we obtain the equation

$$
3(a+b) x-3 y-a^{2}-a b-b^{2}=0
$$

of the locus of all centroids of all tangential triangles in such a pencil. Obviously, the obtained curve is a line parallel to the line $A B$ (Figure 2). So we have proved the following theorem:

THEOREM 2.5. The curve of centroids of all the tangential triangles in the pencil of triangles having the same circumscribed circle is a line in the isotropic plane.

Finally, to obtain the equations of the symmedians, first we need the equations of the angle bisectors of the tangential triangle $A_{t} B_{t} C_{t}$ and those are

$$
\begin{aligned}
& y=(b+c) x-\frac{b^{2}+c^{2}}{2}, \\
& y=(a+c) x-\frac{a^{2}+c^{2}}{2}, \\
& y=(a+b) x-\frac{a^{2}+b^{2}}{2}
\end{aligned}
$$

in the vertices $A_{t}, B_{t}$ and $C_{t}$, respectively. The angle with the vertex $A_{t}$ formed by the median (2.20) and the angle bisector (2.24) must be the same as the angle between the angle bisector (2.24) and the symmedian through the vertex $A_{t}$. It is easy to calculate that the equation of the symmedian through the vertex $A_{t}$ has the equation

$$
y=\frac{2\left(b^{2}+c^{2}-a(b+c)\right)}{-2 a+b+c} x+\frac{\left(b^{2}+c^{2}\right) a-b^{3}-c^{3}}{-2 a+b+c} .
$$

The equations of symmedians through the vertices $B_{t}$ and $C_{t}$ are obtained analogously. All three of them intersect in the same point, the symmedian center of the triangle $A_{t} B_{t} C_{t}$. And once again, we obtain the triangle pencil by moving the point $C$ and eliminating $c$ from any two of three symmedian equations, we obtain the equation of the locus of all symmedian centers of all the triangles in the pencil:

$$
\begin{gathered}
-16\left(a^{3}+2 a^{2} b+2 a b^{2}+b^{3}\right) x^{3}+8\left(5 a^{2}+8 a b+5 b^{2}\right) x^{2} y \\
-36(a+b) x y^{2}+12 y^{3}+4\left(6 a^{4}+7 a^{3} b+10 a^{2} b^{2}+7 a b^{3}+6 b^{4}\right) x^{2} \\
-2\left(19 a^{3}+17 a^{2} b+17 a b^{2}+19 b^{3}\right) x y+\left(17 a^{2}+2 a b+17 b^{2}\right) y^{2} \\
-6\left(2 a^{5}+a^{4} b+3 a^{3} b^{2}+3 a^{2} b^{3}+a b^{4}+2 b^{5}\right) x \\
+\left(9 a^{4}+2 a^{3} b+14 a^{2} b^{2}+2 a b^{3}+9 b^{4}\right) y \\
+2 a^{6}+3 a^{4} b^{2}+2 a^{3} b^{3}+3 a^{2} b^{4}+2 b^{6}=0 .
\end{gathered}
$$


This is a curve of order 3 (Figure 2), therefore we have proved the following theorem:

THEOREM 2.6. The curve of symmedian centers of all the tangential triangles in the pencil of triangles having the same circumscribed circle is a curve of order 3 in the isotropic plane.

The curve of symmedian centers (2.28) has two conjugate-imaginary intersections and one real intersection with the absolute line. The real intersection is a point in which the bisector of the lines $A C_{t}$ and $B C_{t}$ given by (2.26) intersects the absolute line. This curve is a rational cubic having a double point in $C_{t}=\left(\frac{a+b}{2}, a b\right)$, which is parallel to the midpoint of the line segment $A B$. It is easy to check that the fixed lines $A C_{t}$ and $B C_{t}$ are the tangents at $C_{t}$, while the line $y=(a+b) x-\frac{3 a^{2}-2 a b+3 b^{2}}{4}$ is the tangent at its point at infinity.

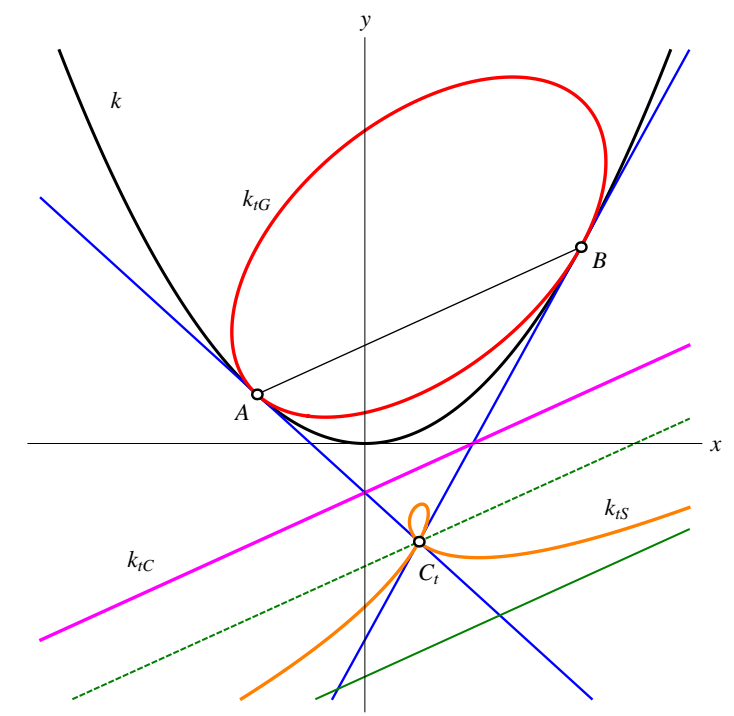

Figure 2. The curve of centroids $k_{t C}$, curve of Gergonne points $k_{t G}$ and curve of symmedian centers $k_{t S}$ for all the tangential triangles in the pencil of triangles with the same circumscribed circle $k$

\section{REFERENCES}

[1] J. Beban-Brkić, V. Volenec, Z. Kolar-Begović and R. Kolar-Šuper, On Gergonne point of the triangle in isotropic plane, Rad Hrvat. Akad. Znan. Umjet. Mat. Znan. 17 (2013), 95-106. 
[2] E. Jurkin, Automorphic inversion and circular quartics in isotropic plane, KoG 12 (2008), 19-26.

[3] E. Jurkin,, Circular quartics in the isotropic plane generated by projectively linked pencils of conics, Acta Math. Hung. 130 (2011), 35-49.

[4] Z. Kolar-Begović, R. Kolar-Šuper, J. Beban-Brkić and V. Volenec, Symmedians and symmedian center of the triangle in an isotropic plane, Math. Pannon. 17 (2006), 287-301.

[5] R. Kolar-Šuper, Z. Kolar-Begović, V. Volenec and J. Beban-Brkić, Metrical relationships in a standard triangle in an isotropic plane, Math. Commun. 10 (2005), 149-157.

[6] H. Sachs, Ebene Isotrope Geometrie, Wieweg, Braunschweig, 1987.

[7] G. Salmon, A Treatise of the Higher Plane Curves: Intended as a Sequel to a Treatise on Conic Section, Chelsea Publishing Company, New York, 1879.

\section{Krivulje težišta, Gergonneovih točaka i sjecišta simedijana pramenova trokuta u izotropnoj ravnini}

\section{Mirela Katić Žlepalo i Ema Jurkin}

SAŽETAK. U ovom radu promatramo pramen trokuta $u$ izotropnoj ravnini koji se sastoji od svih trokuta s istom opisanom kružnicom i proučavamo krivulje težišta, Gergonneovih točaka i sjecišta simedijana takvog pramena. Nadalje, proučavamo i krivulje težišta, Gergonneovih točaka i sjecišta simedijana tangencijalnih trokuta takvog pramena.

Mirela Katić Žlepalo

Department of Civil Engineering

Zagreb University of Applied Sciences

10000 Zagreb, Croatia

E-mail: mkatic@tvz.hr

Ema Jurkin

Faculty of Mining, Geology and Petroleum Engineering

University of Zagreb

10000 Zagreb, Croatia

E-mail: ema.jurkin@rgn.hr

Received: 23.8.2017.

Revised: 23.2.2018. 